\title{
Eigenspectrum calculation of the non-Hermitian $O(a)$-improved Wilson-Dirac operator using the Sakurai-Sugiura method
}

\author{
H. Suno ${ }^{* a, b}$, Y. Nakamura ${ }^{a}$, K.-I. Ishikawa $^{c}$, Y. Kuramashi ${ }^{a, d, e}$, Y. Futamura ${ }^{f}$, A. \\ Imakura $^{f}$, and T. Sakurai ${ }^{f}$ \\ ${ }^{a}$ RIKEN Advanced Institute for Computational Science, Kobe, Hyogo 650-0047, Japan \\ ${ }^{b}$ RIKEN Nishina Center for Accelerator-Based Science, Wako, Saitama 351-0198, Japan \\ ${ }^{c}$ Department of Physical Science, Hiroshima University, Higashi-Hiroshima, Hiroshima \\ 739-8526, Japan \\ ${ }^{d}$ Center for Computational Science, University of Tsukuba, Tsukuba, Ibaraki 305-8577, Japan \\ ${ }^{e}$ Faculty of Pure and Applied Science, University of Tsukuba, Tsukuba, Ibaraki 305-8571, Japan \\ ${ }^{f}$ Department of Computer Science, University of Tsukuba, Tsukuba, Ibaraki 305-8573, Japan \\ E-mail: sunodriken.ip
}

\begin{abstract}
We are developing a computer code for calculating eigenvalues and eigenvectors of non-Hermitian matrices arising in lattice QCD. We introduce here the Sakurai-Sugiura (SS) method, which is based on a contour integral, allowing us to obtain desired eigenvalues located inside a given contour of the complex plane. We apply our implementation to calculating low-lying eigenvalues of the non-Hermitian $O(a)$-improved Wilson-Dirac operator. We are particularly interested in such cases as finding the lowest eigenvalues to be equal or close to zero in the complex plane. Our implementation is tested for the free-case Wilson-Dirac operator, while eigenvalue calculations are also carried out using different sets of gauge configurations obtained in quenched approximation and in a full QCD simulation. We consider the lattice sizes $8^{3} \times 16$ and $96^{4}$.
\end{abstract}

The 33rd International Symposium on Lattice Field Theory

14 -18 July 2015

Kobe International Conference Center, Kobe, Japan*

\footnotetext{
* Speaker.
} 


\section{Introduction}

The determinant of the Wilson-Dirac operator, $\operatorname{det} D$, plays an important role in lattice QCD. In general, the determinant of an $\mathscr{L} \times \mathscr{L}$ matrix $D$ can be written, in terms of its eigenvalues $\lambda_{l}$, as

$$
\operatorname{det} D=\prod_{l=1}^{\mathscr{L}} \lambda_{l}
$$

so that all the information about the Wilson fermion determinant, or the fermion measure, is concentrated in the eigenspectrum. This makes the eigenspectrum calculation of the Wilson-Dirac operator an interesting subject. Because the eigenspectrum possesses the vertical and horizontal symmetries and that the complex eigenvalues are therefore always paired, the determinant can be further expressed in the form:

$$
\operatorname{det} D=\prod_{\lambda_{l} \in \mathbb{R}} \lambda_{l} \prod_{\lambda_{l^{\prime}} \in \mathbb{C}}\left|\lambda_{l^{\prime}}\right|^{2}
$$

We are thus particularly interested in calculating several low-lying real eigenvalues of the WilsonDirac operator, since the sign problem may occur due to the real, negative eigenvalues.

In this work, we develop exploratorily a computer code for calculating the low-lying eigenspectrum of the Wilson-Dirac operator. We adopt here the Sakurai-Sugiura method, which is based on contour integrals, allowing us to calculate eigenvalues located in a given domain of the complex plane as well as the associated eigenvectors. Our computer code will be applied to calculating low-lying eigenvalues of the non-Hermitian $O(a)$-improved Wilson-Dirac operator. We consider the spatiotemporal lattice sizes $8^{3} \times 16$ and $96^{4}$, amounting to the matrix order of 98,304 and $1,019,215,872$, respectively. Eigenvalue calculations will be performed using gauge field configurations for the free case, those generated in quenched approximation as well as those generated by a full QCD simulation, focusing on such cases as finding the low-lying eigenvalues to be localized very close to zero in the complex plane.

\section{Sakurai-Sugiura method for the Wilson-Dirac operator}

The lattice QCD is defined on a hypercubic four-dimensional lattice of finite extent expressed as $L_{x} \times L_{y} \times L_{z} \times L_{t}$ with $L_{x, y, z}$ being the three-dimensional spatial extent and $L_{t}$ the temporal one. The lattice spacing is set to unity for notational convenience. The fields are defined on the sites $n$ with periodic boundary conditions. We define two types of fields on the lattice. One is the gauge field represented by $U_{\mu}(n)^{a, b}$ with $\mu=1,2,3,4$ (corresponding respectively to $\left.x, y, z, t\right)$ and $a, b=1,2,3$, which is a $3 \times 3 \mathrm{SU}(3)$ matrix assigned on each link. The other is the quark field $q(n)_{\alpha}^{a}$ which resides on each site carrying the Dirac index $\alpha=1,2,3,4$ and the color index $a=1,2,3$. The $O(a)$-improved Wilson-Dirac operator is written as

$$
\begin{aligned}
D_{\alpha, \beta}^{a, b}(n, m)= & \delta_{\alpha, \beta} \delta^{a, b} \delta(n, m)-\kappa \sum_{\mu=1}^{4}\left[\left(1-\gamma_{\mu}\right)_{\alpha, \beta}\left(U_{\mu}(n)\right)^{a, b} \delta(n+\hat{\mu}, m)\right. \\
& \left.+\left(1+\gamma_{\mu}\right)_{\alpha, \beta}\left(\left(U_{\mu}(m)\right)^{b, a}\right)^{*} \delta(n-\hat{\mu}, m)\right] \\
& +\kappa c_{\mathrm{SW}} \sum_{\mu, v=1}^{4} \frac{i}{2}\left(\sigma_{v \mu}\right)_{\alpha, \beta}\left(F_{v \mu}(n)\right)^{a, b} \delta(n, m)
\end{aligned}
$$


where $\hat{\mu}$ denotes the unit vector in the $\mu$ direction. $\kappa$ is the hopping parameter, and the coefficient $c_{\mathrm{SW}}$ is a parameter to be adjusted for the $O(a)$ improvement. The Eulidean gamma matrices are defined in terms of the Minkowski ones in the Bjorken-Drell convention: $\gamma_{j}=-i \gamma_{B D}^{j}(j=1,2,3)$, $\gamma_{4}=\gamma_{B D}^{0}, \gamma_{5}=\gamma_{B D}^{5}$, and $\sigma_{\mu \nu}=\frac{1}{2}\left[\gamma_{\mu}, \gamma_{v}\right]$. The explicit representation for $\gamma_{1,2,3,4,5}$ are given in Ref. [四], together with the expression for the field strength $F_{\mu v}(n)$ in terms of the gauge field $U_{\mu}(n)$. The $O(a)$-improved Wilson-Dirac operator defined in Eq. (ㅁ. $(\mathbb{d})$ is a sparse, complex nonHermitian square matrix $D \in \mathbb{C}^{\mathscr{L} \times \mathscr{L}}$, where only 51 out of $\mathscr{L}=L_{x} \times L_{y} \times L_{z} \times L_{t} \times 3 \times 4$ entries in each row have nonzero values.

In this work, we consider an eigenproblem

$$
D x_{l}=\lambda_{l} x_{l},(l=1,2, \ldots, \mathscr{L}),
$$

where $\lambda_{l}$ and $x_{l}$ are eigenvalues and eigenvectors, respectively. In order to extract eigenpairs $\left(\lambda_{l}, x_{l}\right)$ from the matrix $D$ in Eq. ( 22$]$, we adopt the Sakurai-Sugiura method, proposed in Refs. [ [य, [], 团]. This method is based on contour integrals, allowing us to calculate eigenvalues located in a given domain of the complex plane, together with the associated eigenvectors. In this method, we first define matrices $S_{k} \in \mathbb{C}^{\mathscr{L} \times L}$ as

$$
S_{k} \equiv \frac{1}{2 \pi i} \oint_{\Gamma} z^{k}(z I-D)^{-1} V d z, k=0,1, \ldots, M-1 .
$$

Here, $\Gamma$ is a positively oriented closed curve in the complex plane inside which we seek for eigenvalues, and $M$ denotes the maximum moment degree. The matrix $V \in \mathbb{C}^{\mathscr{L} \times L}$, called source matrix, contains $L$ column vectors $V=\left[v_{1}, v_{2}, \ldots, v_{L}\right]$, and we take a random vector for each of these source vectors. We assume that $\Gamma$ is given by a circle and the integration is evaluated via a trapezoidal rule on $\Gamma$. By designating the center and the radius of the circle as $\gamma$ and $\rho$, respectively, and by defining the quadrature points as $z_{j}=\gamma+\rho e^{2 \pi i(j-1 / 2) / N}, j=1,2, \ldots, N$, we approximate the integral in Eq. (L.3) via an $N$-point trapezoidal rule:

$$
S_{k} \approx \hat{S}_{k} \equiv \frac{1}{N} \sum_{j=1}^{N} z_{j}^{k}\left(z_{j} I-D\right)^{-1} V .
$$

We then carry out the singular value decomposition for the matrix $\hat{S}=\left[\hat{S}_{0}, \hat{S}_{1}, \ldots, \hat{S}_{M-1}\right] \in \mathbb{C}^{\mathscr{L} \times L M}$ as follows

$$
\begin{aligned}
\hat{S} & =\tilde{Q} \Sigma W, \\
\tilde{Q} & =\left[q_{1}, q_{2}, \ldots, q_{L M}\right] \in \mathbb{C}^{\mathscr{L} \times L M}, \\
\Sigma & =\operatorname{diag}\left(\sigma_{1}, \sigma_{2}, \ldots, \sigma_{L M}\right) .
\end{aligned}
$$

We next determine the numerical rank $m$ of the matrix $\hat{S}$. The value of $m$ is fixed as the number of singular values satisfying $\sigma_{i}>\delta$, with $\delta$ being the threshold for determining the numerical rank and usually set to $\delta=10^{-12}$. The original eigenproblem is transformed to a smaller eigenproblem via the transformation matrix $Q=\left[q_{1}, q_{2}, \ldots, q_{m}\right]$, with only the first $m$ column vectors from $\tilde{Q}$ are incorporated. We finally solve the smaller eigenequation $Q^{\mathrm{H}} D Q u_{l}=\mu_{l} u_{l}$ and obtain an approximation to the eigenpairs $\lambda_{l} \approx \mu_{l}$ and $x_{l} \approx Q u_{l}$. 
As can be seen from Eq. (2.4), the Sakurai-Sugiura method produces a subspace with the matrix basis involving the inverses of the matrices $\left(z_{j} I-D\right)$. The matrix inversion can be performed solving the shifted linear equations

$$
\left(z_{j} I-D\right) y_{j l}=v_{l} .
$$

There exist several implementations based on direct methods such as LAPACK and MUMPS libraries. These implementations, however, are hardly applicable to such linear problems as arising in lattice QCD due to their large matrix sizes, and some iterative methods are desirable to solve such large sparse linear equations. In this work, we implement exploratorily the BiCGStab algorithm as is presented in Algorithm $\square$. The BiCGStab algorithm will be found to converge very slowly, suffering from the ill-condition problem of the shifted linear equations. We choose, however, to employ the solution vectors as are obtained from a sufficiently large number of BiCGStab iterations. Code development is carried out based on the lattice QCD simulation program LD-

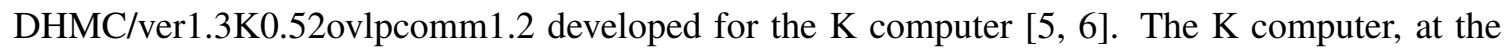
RIKEN Advanced Institute for Computational Science, consists of 82944 computational nodes and $5184 \mathrm{I} / \mathrm{O}$ nodes connected by the so-called "Tofu" network, providing 11.28 Pflops of computing capability. The Tofu network topology is six-dimensional one with 3D-mesh times 3D-torus shape. Each node has a single $2.0 \mathrm{GHz}$ SPARC64 VIIIfx processor equipping 8 cores with SIMD enabled 256 registers, $6 \mathrm{MB}$ shared $\mathrm{L} 2$ cache and $16 \mathrm{~GB}$ of memory. The $\mathrm{L} 1$ cache sizes per each core are $32 \mathrm{~KB} / 2 \mathrm{WAY}$ (instruction) and 32KB/2WAY (data).

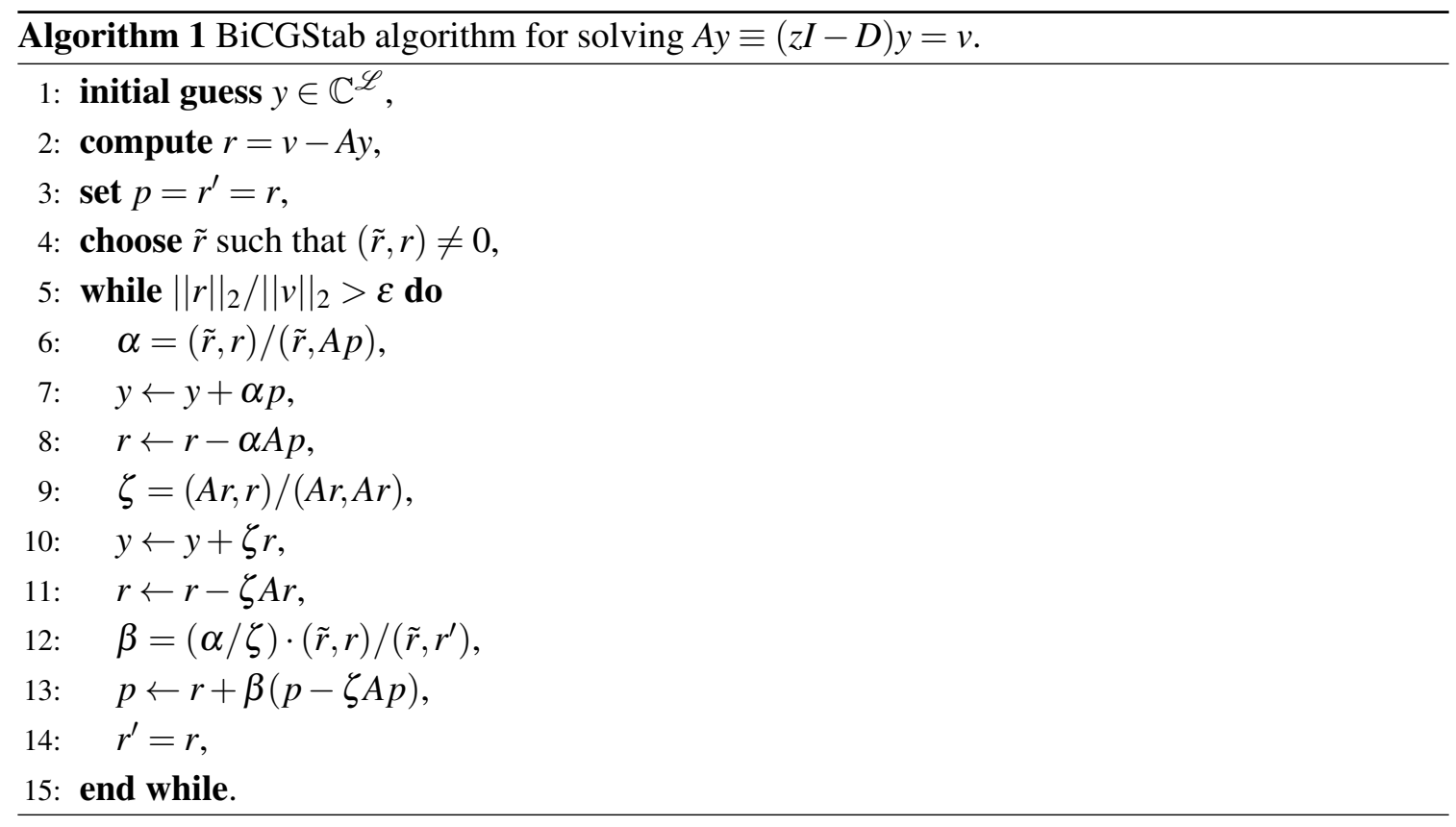

\section{Simulation results}

Our implementation is tested here for the free-case Wilson-Dirac operator, of which the eigenspectrum can be analytically obtained. For the free case, $\left(U_{\mu}(n)\right)^{a, b}=\delta_{a b}$, the Wilson-Dirac action, 
in the momentum space $\left(\psi(x)=\int d k \exp (i k x) \tilde{\psi}(k)\right)$, turns out to be

$$
\tilde{D}(k)=1-\kappa \sum_{\mu=1}^{4}\left[2 \cos \left(k_{\mu}\right)-2 i \gamma_{\mu} \sin \left(k_{\mu}\right)\right]
$$

Since the allowed momentum components must be the discrete elements of the Brillouin zone $\left(k_{\mu}=\frac{2 \pi}{L_{\mu}} l_{\mu}\right.$ ), we then obtain the expression for the free Wilson-Dirac eigenvalues as

$$
\lambda_{\left\{l_{\mu}\right\}}^{\mathrm{free}}=1-2 \kappa\left[\sum_{\mu=1}^{4} \cos \left(\frac{2 \pi}{L_{\mu}} l_{\mu}\right) \pm i \sqrt{\sum_{\mu=1}^{4} \sin ^{2}\left(\frac{2 \pi}{L_{\mu}} l_{\mu}\right)}\right], l_{\mu}=0,1, \ldots, L_{\mu}-1 .
$$

Each set of the numbers $\left\{l_{\mu}\right\}$ correspond to 12 eigenvalues, 2 sets of 6 multiple eigenvalues with the plus and minus signs in Eq. (B.2). Note also that for the hopping parameter $\kappa=1 / 8$, the minimum eigenvalue coincide with the origin, $z=0$.

The left panel of Fig.W shows the eigenvalues of the free-case Wilson-Dirac operator calculated by the SS method for the lattice size $8^{3} \times 16$ and the hopping parameter $\kappa=1 / 8$. We have used the number of quadrature $N=32$, the number of source vector $L=64$ and the maximum moment degree $M=16$. We notice three sets of eigenvalues inside the integration contour of which the quadrature points $z_{j}$ are indicated by asterisks. Each of these three sets contains 12 multiple eigenvalues, so that in total 36 eigenvalues are found inside the integration contour. The BiCGStab algorithm used for solving the shifted linear equation ([2.8) is found to converge very slowly for some quadrature points $z_{j}$, due to the ill-condition problem: for the quadrature point the most on the right-hand side $z_{1}$, the residual $\|r\|_{2} /\|v\|_{2}$ decreases only to about $10^{-8} \sim 10^{-10}$ with 1000 BiCGStab iterations, while it decreases less than $10^{-14}$ with about 200 BiCGStab iterations for the quadrature point the most on the left-hand side $z_{17}$. However, using the solution vectors obtained with about 1000 iterations, we have been able to obtain the above eigenvalues accurately with the relative residual norms about $10^{-7}$. Our eigenvalues obtained by the SS method are visually indistinguishable from those obtained from the analytical expression in Eq. (3.2). The right panel of Fig. $\Pi$ shows the same results but for the larger lattice size, $96^{4}$. We have used the parameters for the SS method $(N, L, M)=(32,128,16)$. Here, inside the integration contour, we have found one set of 12 multiple eigenvalues at the origin and 2 sets of 36 multiple eigenvalues above and below the origin, 84 eigenvalues in total. The relative residual norms have been found to be around $5 \times 10^{-4}$. These eigenvalues calculated by the SS method are also shown to be indistinguishable from those from the analytical expression in Eq. (B.2). Note that, we set $L=128$ since it must be greater than or equal to the maximum multiplicity of the eigenvalues in $\Gamma$. If there is no multiplicity, we usually set a small value to $L$ e.g. $L=8$.

We have also carried out eigenvalue calculations with a sample of gauge field configurations generated in quenched approximation. We generate these configurations on a $8^{3} \times 16$ lattice with the Iwasaki gauge action $\beta=1.9$ using the lattice QCD program LDDHMC/ver1.3K0.52ovlpcomm1.2 [1], 目]. The left panel of Fig. $\square$ shows the eigenvalues of the $O(a)$-improved Wilson-Dirac operator with these gauge configurations in quenched approximation for the parameters $\kappa=0.1493$ and $c_{\mathrm{SW}}=1.6$. These values of $\kappa$ and $c_{\mathrm{SW}}$ have been chosen so that the minimum real eigenvalue is close to the origin in the complex plane. We have used the parameters for the SS method 

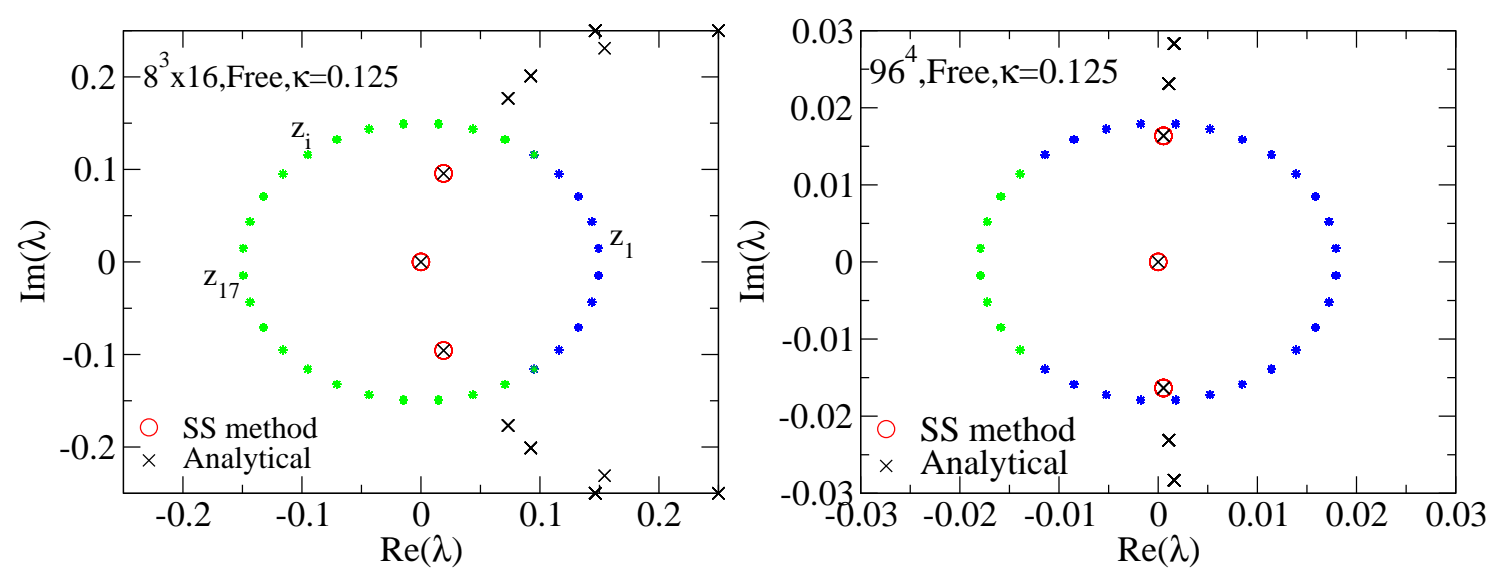

Figure 1: Left: Eigenvalues of the free-case Wilson-Dirac operator in a $8^{3} \times 16$ lattice for the hopping parameter $\kappa=1 / 8$. The eigenvalues obtained from the SS method are indicated as red circles, those obtained from the analytical expression are indicated as black crosses. Green (blue) asterisks indicate the quadrature points $z_{j}$ for which the BiCGStab algorithm converges (does not converge) to less than $10^{-14}$ of the relative residuals norms with 1000 iterations. Right: The same as in the left panel, but for the lattice size $96^{4}$.

$(N, L, M)=(32,96,16)$. The relative residual norms of the eigenvalues have been found to be around $10^{-5}$.

Finally, we have performed eigenvalue calculations using a set gauge field configurations generated in a full QCD calculation. These gauge field configurations are generated by a $2+1$ flavor QCD simulation near the physical point on a $96^{4}$ lattice [ $[0]$ with the hopping parameters $\left(\kappa_{\mathrm{ud}}, \kappa_{\mathrm{s}}\right)=(0.126117,0.124700)$ and $c_{\mathrm{SW}}=1.110$. The other details are given in Ref. [四]. The right panel of Fig. $\square$ shows the eigenvalues calculated by the SS method for the hopping and improvement parameters $\kappa=0.126117$ and $c_{\mathrm{SW}}=1.110$. We have used the parameters for the SS method $(N, L, M)=(32,16,16)$, and the maximum number of BiCGStab iterations 30000 for solving the shifted linear equations. The relative residual norms of the eigenvalues have been found to be around $5 \times 10^{-4}$.

The above eigenvalue calculations have been performed on the K computer, using 16 nodes for the lattice size $8^{3} \times 16$ and 16,384 nodes for the lattice size $96^{4}$. Almost the whole computer time is consumed in the multiplication of the quark field by the Wilson-Dirac operator. The time spent by each matrix-vector multiplication is found to be $1.29 \times 10^{-3} \mathrm{sec}$ for the lattice size $8^{3} \times 16$, and $5.5 \times 10^{-3} \mathrm{sec}$ for the lattice size $96^{4}$. For the $8^{3} \times 16$ free case, the elapsed time amounts to 2,160 sec with about $1.67 \times 10^{6}$ matrix-vector multiplications being performed. For the $96^{4}$ free case, the elapsed time is $21,500 \mathrm{sec}$ with about $3.93 \times 10^{6}$ matrix-vector multiplications. For the $8^{3} \times 16$ quenched-approximation case, the elapsed time is found to be $3,480 \mathrm{sec}$ with about $3.01 \times 10^{6}$ matrix-vector multiplications, while for the $96^{4}$ Full QCD case, we have found the elapsed time to be $65,300 \mathrm{sec}$ with $1.27 \times 10^{7}$ matrix-vector multiplications.

\section{Summary}

In this work, we have exploratorily developed a computer code for calculating eigenvalues and eigenvectors of non-Hermitian matrices arising in lattice QCD, using the Sakurai-Sugiura method. 

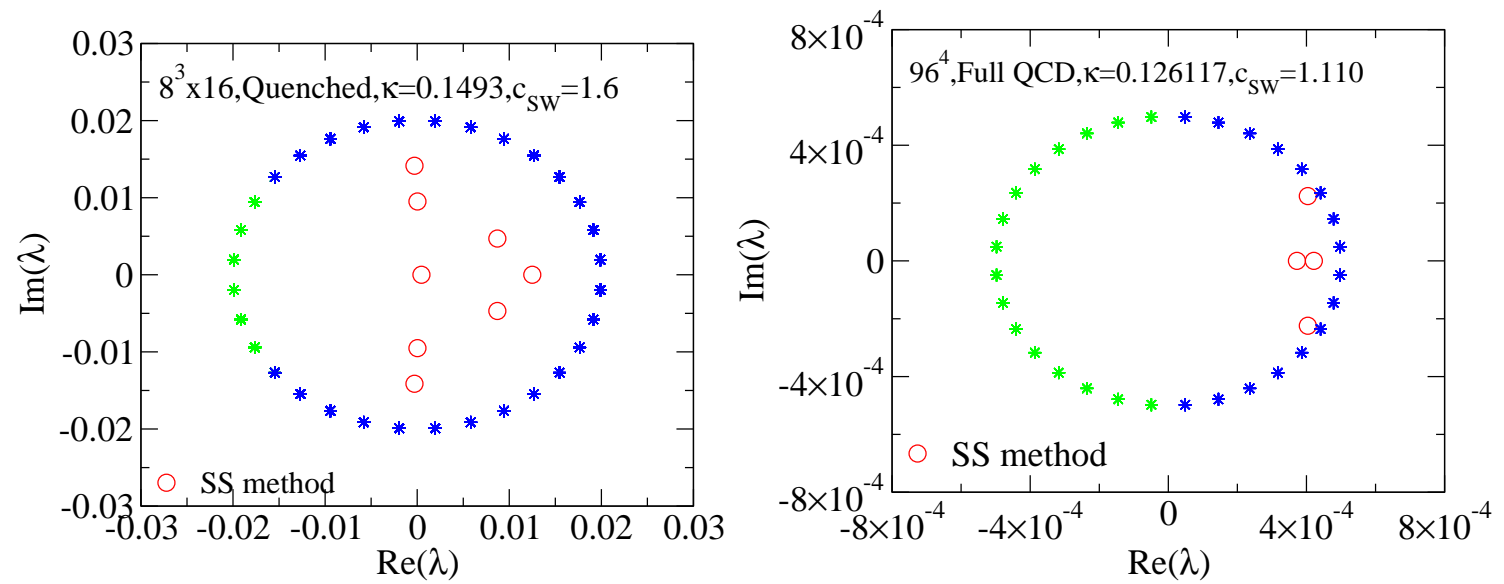

Figure 2: Left: Eigenvalues of the $O(a)$-improved Wilson-Dirac operator in a $8^{3} \times 16$ lattice for the hopping parameter $\kappa=0.1493$ and the improvement parameter $c_{\mathrm{SW}}=1.6$, with gauge field configurations generated in quenched approximation. The eigenvalues obtained from the SS method are indicated as red circles. Green (blue) asterisks indicate the quadrature points $z_{j}$ for which the BiCGStab algorithm converges (does not converge) to less than $10^{-14}$ of the relative residuals norms with 1000 iterations. Right: Eigenvalues of the $O(a)$-improved Wilson-Dirac operator in a $96^{4}$ lattice for the hopping parameter $\kappa=0.126117$ and the improvement parameter $c_{\mathrm{SW}}=1.110$, with gauge field configurations generated in a full QCD simulation. The eigenvalues obtained from the SS method are indicated as red circles. Green (blue) asterisks indicate the quadrature points $z_{j}$ for which the BiCGStab algorithm converges (does not converge) to less than $10^{-14}$ of the relative residuals norms with 30000 iterations.

We have applied our implementation to calculating low-lying eigenvalues of the $O(a)$-improved Wilson-Dirac operator with gauge field configurations for the free case and those generated in quenched approximation and in full QCD. Eigenvalues have been obtained with relative residual norms from about $10^{-7}$ to $5 \times 10^{-4}$, with the accuracy being limited by the slow convergence of the BiCGStab algorithm for solving the shifted linear equations. A more efficient shifted linear equation solver is desirable in order to improve the accuracy of these eigenvalues. We are actually carrying out implementation of a Krylov subspace iterative method specifically for solving shifted linear systems $[$ [ $]$.

\section{References}

[1] T. Sakurai, H. Tadano, and Y. Kuramashi, Comp. Phys. Comm. 181, 113 (2010).

[2] T. Sakurai and H. Sugiura, J. Comput. Appl. Math. 159, 119 (2003).

[3] T. Sakurai, Y. Futamura, and H. Tadano, J. Algorithms Comput. Technol. 7, 249 (2013).

[4] A software named "z-Pares" is available at the web site http://zpares.cs.tsukuba.ac.jp.

[5] PACS-CS Collaboration, S. Aoki et al., Phys. Rev. D 79, 034503 (2009).

[6] T. Boku et al., PoS LATTICE2012 188 (2012).

[7] N. Ukita et al., PoS LATTICE2015, 194 (2015).

[8] M. Ogasawara, H. Tadano, T. Sakurai, and S. Itoh, J. Soc. Ind. App. Math. 14, 193 (2004) (in japanese). 